\title{
Using the principles of 'same day emergency care' in our new acute medical unit
}

\author{
Authors: Shaznin Visanji, ${ }^{A *}$ Holly Lyne, ${ }^{\mathrm{A}}$ Alexandra Phillips, ${ }^{\mathrm{A}}$ Hazel Gilbert, ${ }^{\mathrm{A}}$ Zachary Ferguson, ${ }^{\mathrm{A}}$ \\ Amber Hawksley ${ }^{A_{*}}$ and Robert Barker ${ }^{A}$
}

\section{Introduction}

In January 2019, Barnet Hospital opened the new acute medical unit (AMU), which uses the objectives and aims set out by NHS Improvement 'same-day emergency care' (SDEC). ${ }^{1}$ The ambulatory emergency clinic (AEC) forms part of the new AMU, as a clinic for those patients 'fit to sit', to avoid unnecessary hospital admissions in clinically stable, ambulatory patients.

The principles of SDEC were used to answer the question: 'Why does it take so long for general practitioner (GP) expected patients to be discharged?'

The objective was to determine any factors that lead to a delay in assessment, investigation, management and, ultimately, discharge.

The overall aims of the project are to reduce the time a stable patient spends in the department and to re-triage stable patients to AEC.

\section{Methods}

Baseline data were collected in October 2018, prior to the new unit opening, on 'GP referred' patients that were discharged home the same day. Data were collected on time to triage, first medical review, senior decision maker and decision to discharge.

Plan, do, study, act cycles were used throughout the project and data were collected prospectively from January 2019.

$>$ Moving the twilight registrar onto the unit.

$>$ Single point of triage.

$>$ Transferring patients from the emergency department (ED) with a GP letter.

$>$ Access to assessment trolleys.

\section{Results and discussion}

By improving the way patients are processed, the time spent in the department was significantly reduced.

Access to an early senior decision maker allows for prompt decision making along all parts of the patients' pathway, allowing for early decision to discharge.

Implementing an additional senior decision maker improved our average time to senior review from 3 hours 19 minutes to 2 hours
56 minutes and subsequently the decision to discharge from 3 hours 28 minutes to 2 hours 30 minutes.

Having a single point of triage means that patients are processed efficiently at triage and packaged ready to be seen by a doctor. At the point of arrival all patients have observations done, a set of bloods and any other investigations needed. Having a unified triage process improved the time to triage from 39 minutes to 20 minutes.

Access to assessment trolleys allowed the unit to accept and manage those patients more acutely unwell direct from the GP without the need for an ED admission.

The burden on the medical take was also significantly reduced; $50 \%$ of the GP expected patients were seen by the medical take via the ED with $9 \%$ being reviewed in AEC; after the unit opened $47 \%$ were seen in AEC with only $7 \%$ being reviewed in ED.

\section{Conclusion}

By improving the efficiency in the patients' pathway, we successfully used the principles set out by the SDEC guidelines. The time a patient spends in the department has significantly reduced. The unit has also helped the reduce the burden on ED by accepting patients with a GP letter directly.

\section{Conflicts of interest}

None declared.

\section{Reference}

1 NHS Improvement, Ambulatory Care Network. Same-day emergency care: clinical definition, patient selection and metrics. NHS, 2019. 